\title{
EFEITO DE DIFERENTES TRATAMENTOS NO CRESCIMENTO DE MUDAS DE PALMEIRA-REAL-AUSTRALIANA
}

\author{
Eliséo Soprano ${ }^{1}$, Fábio Martinho Zambonim ${ }^{1}$, Airton Rodrigues Salerno ${ }^{1}$, Terezinha Catarina \\ Heck $^{1}$, Alexandre Visconti ${ }^{1}$, Alessandro Borini Lone ${ }^{1}$
}

${ }^{1}$ Empresa de Pesquisa Agropecuária e Extensão Rural de Santa Catarina (EPAGRI) - Estação Experimental de Itajaí, E-mail: esoprano@outlook.com,_zambonim@epagri.sc.gov.br,_ salerno@epagri.sc.gov.br, heck@epagri.sc.gov.br, visconti@epagri.sc.gov.br, alessandrolone@epagri.sc.gov.br

\section{RESUMO}

O Brasil é considerado um dos maiores produtores e consumidores mundiais de palmito. $\mathrm{O}$ cultivo comercial de palmeira-real-australiana (Archontophoenix spp.) para produção de palmito destaca-se nos Estados de Santa Catarina e Paraná. O objetivo foi identificar a resposta na produção de matéria seca da parte aérea de mudas de palmeira-real-australiana à aplicação de diferentes doses, fontes de nutrientes e $\mathrm{pH}$ no solo. Os tratamentos foram concebidos de uma matriz experimental baconiana, onde foram avaliados níveis de $\mathrm{pH}$, doses de Nitrogênio, Fósforo, Potássio e de cama de aviário, totalizando 21 tratamentos em blocos casualizados com quatro repetições. Pode-se concluir que as doses de máxima eficiência técnica que garantiram as melhores respostas em produção de matéria seca da parte aérea de mudas de palmeira-real-australiana foram 100,3 mg de N, 401,7 mg de P e 215,5 mg de K por kg de solo e pH de 4,94.

Palavras-chave: Nutrição mineral, Palmito, Produção de mudas

\section{EFFECT OF DIFFERENT TREATMENTS IN AUTRALIAN ROYAL PALM SEEDLINGS GROWTH}

\begin{abstract}
Brazil is considered one of the biggest world producers and consumers of heart palm. The commercial cultivation of autralian royal palm (Archontophoenix spp.) for heart palm production stands out in Santa Catarina and Paraná states. The aim was to identify the answer in the production of dry matter of the aerial part of australian royal palm seedlings to the application of different doses, nutrient sources and $\mathrm{pH}$ in the soil. The treatments were designed in a Baconian experimental matrix, which were evaluated $\mathrm{pH}$ levels, levels of Nitrogen, Phosphorus and Potassium and poultry litter, totaling 21 treatments in a randomized block design with four
\end{abstract}


replications. It can be conclude that the maximum technical efficiency doses that ensured the best answers in dry matter production of the aerial part of australian royal palm seedlings were 100.3 $\mathrm{mg}$ of $\mathrm{N}, 401.7 \mathrm{mg}$ to $215.5 \mathrm{mg}$ of $\mathrm{P}$ and $\mathrm{K}$ per $\mathrm{kg}$ of soil and $\mathrm{pH} 4.94$.

Keywords: Heart palm, Mineral nutrition, Seedling production

\section{INTRODUÇÃO}

O palmito é um alimento obtido da extremidade apical do estipe de determinadas espécies de palmeiras, sendo composto por folhas em formação. O Brasil é considerado um dos maiores produtores e consumidores mundiais de palmito (SILVA \& BANDEIRA, 2008). No âmbito nacional, a atividade de industrialização de palmito em conserva iniciou-se em Santa Catarina em meados da década de 1960, alicerçada no extrativismo de povoamentos naturais de palmeira-juçara (Euterpe edulis). A partir do início dos anos 70 desenvolveu-se mais fortemente no Pará, por meio da exploração de povoamentos naturais de açaizeiro (Euterpe oleracea) (RODRIGUES \& DURIGAN, 2008).

Segundo Rodrigues \& Durigan (2007), até 1993 praticamente toda a produção de palmito provinha do extrativismo (cerca de 98\%). A partir de 1994 houve o incremento das áreas cultivadas, atingindo $74 \%$ da produção em 2002, evidenciando a tendência do setor em migrar de uma atividade predominantemente extrativista para outra baseada em cultivos comerciais. Além de Euterpe spp., são cultivadas para produção de palmito no Brasil a pupunheira (Bactris gasipaes), nos Estados de SP e BA, e a palmeira-real-australiana (Archontophoenix spp.), nos Estados de SC e PR (RODRIGUES \& DURIGAN, 2008). Essa última espécie desenvolve-se bem a pleno sol nas várzeas do litoral catarinense, estando a cultura em expansão nessa região. Em relação ao manejo da adubação da palmeira-real-australiana para produção de palmito, existem poucas indicações técnicas disponíveis e muitas são preliminares.

A adubação permite elevar o teor dos nutrientes no solo a níveis considerados adequados para as culturas expressarem todo seu potencial produtivo, sempre que os demais fatores não sejam limitantes (BISSANI et al., 2008). O manejo adequado da adubação de determinada cultura demanda, dentre outros fatores, a definição das doses e das fontes de nutrientes a serem utilizados (ANGHINONI \& BAYER, 2004). O objetivo deste trabalho foi identificar a resposta na produção de matéria seca da parte 
aérea de mudas de palmeira-real-australiana à aplicação de diferentes doses e fontes de nutrientes no solo.

\section{MATERIAL E MÉTODOS}

O experimento foi conduzido no período de 07/07/2011 a 08/11/2012 em de casa de vegetação na Estação Experimental de Itajaí (EEI) - EPAGRI, Itajaí, SC. A EEI encontra-se localizada na Latitude $26^{\circ} 57^{\prime}$ 06' Sul e Longitude $48^{\circ} 45^{\prime} 38^{\prime}$ ' W e 10 m acima do nível do mar. O clima da região é Mesotérmico Úmido com verão quente. A temperatura média é de $21^{\circ} \mathrm{C}$ e no inverno, esporadicamente, a temperatura atinge a marca de $10{ }^{\circ} \mathrm{C}$, sendo a ocorrência de geadas rara. As chuvas apresentam um regime mais intenso no verão, variando entre $1.400 \mathrm{~mm}$ a $2.000 \mathrm{~mm}$ anuais. A umidade relativa do ar é de aproximadamente $84 \%$ e a pressão atmosférica média é de 1.010 milibares.

As mudas de palmeira-realaustraliana (Archontophoenix alexandrae) foram cultivadas em vasos plásticos contendo $8 \mathrm{dm}^{3}$ de um Argissolo Vermelho-Amarelo alumínico típico. A camada de solo utilizada foi coletada entre $10 \mathrm{~cm}$ e $30 \mathrm{~cm}$ de profundidade e apresentou as seguintes características químicas: $\mathrm{pH}$ em $\mathrm{H}_{2} \mathrm{O}$ (1:1) 4,1; Índice SMP 5,2; P 1,5 mg L-1 (Mehlich 1); K 19,0 mg L-1 (Mehlich -1); M.O. 10,0g $\mathrm{kg}^{-1} ; \mathrm{Al}(\mathrm{KCl} 1 \mathrm{~N}) 2,6 \mathrm{cmol}_{\mathrm{c}} \mathrm{L}^{-1} ; \mathrm{Ca}(\mathrm{KCl} 1 \mathrm{~N})$ 2,6 $\mathrm{cmolcL}^{-1} ; \mathrm{Mg}(\mathrm{KCl} 1 \mathrm{~N})$ 1,0 $\mathrm{cmol}_{\mathrm{c}} \mathrm{L}^{-1} \mathrm{e}$ Saturação por $\mathrm{Al} 47,7 \%$.

Os tratamentos foram concebidos de uma matriz experimental baconiana (TURRENT, 1979), onde foram avaliados níveis de $\mathrm{pH}$, doses de $\mathrm{N}, \mathrm{P}, \mathrm{K}$ e de cama de aviário, totalizando 21 tratamentos que foram distribuídos em blocos casualizados com quatro repetições (Tabela 1). Os tratamentos foram arranjados de forma que, quando a quantidade de um variasse, as dos outros estariam fixas de acordo com a dose referência (Tratamento 1).

A amostra de solo foi seca ao ar e passada em peneira de malha de $6 \mathrm{~mm}$. Em seguida foram colocadas em vasos de 8 litros revestidos internamente por saco plástico para evitar perdas de nutrientes por lixiviação. As aplicações dos tratamentos foram feitas via sólida para $\mathrm{P}$, cama de aviário e correção do pH e, em solução, para N e K. As fontes de nutrientes foram ureia, superfosfato triplo e cloreto de potássio, para $\mathrm{N}, \mathrm{P}$ e $\mathrm{K}$ respectivamente. Como fonte de material/adubo orgânico utilizou-se cama de aviário de quatro lotes. Como corretivo da acidez do solo foi utilizada uma mistura de $\mathrm{CaCO}_{3}+\mathrm{MgCO}_{3}$ na relação estequiométrica de $4: 1$. A definição da quantidade de corretivo a ser aplicada nos tratamentos níveis de $\mathrm{pH}$ 
foi feita a partir de uma curva de eliminadas, mantendo-se uma, constituindo neutralização.

Após um período de incubação de 30 uma unidade experimental. A irrigação foi feita com água destilada, de acordo com as dias, plantaram-se três mudas por vaso. Após necessidades das plantas e, o controle, através o estabelecimento, duas mudas foram de pesagens periódicas.

Tabela 1. Esquema dos tratamentos aplicados no solo de cultivo de mudas de palmeira-realaustraliana, Itajaí, SC, 2012.

\begin{tabular}{|c|c|c|c|c|c|}
\hline Tratamentos & $\mathrm{N}$ & $\mathrm{P}$ & $\mathrm{K}$ & $\mathrm{pH}$ & Cama de Aviário \\
\hline & - & $\mathrm{mg} \mathrm{kg}^{-1}$ & ـ & & $\mathrm{Mg} \mathrm{ha}^{-1}$ \\
\hline $1 *$ & 100 & 150 & 150 & 6 & 0 \\
\hline 2 & 0 & 150 & 150 & 6 & 0 \\
\hline 3 & 50 & 150 & 150 & 6 & 0 \\
\hline 4 & 150 & 150 & 150 & 6 & 0 \\
\hline 5 & 200 & 150 & 150 & 6 & 0 \\
\hline 6 & 400 & 150 & 150 & 6 & 0 \\
\hline 7 & 100 & 0 & 150 & 6 & 0 \\
\hline 8 & 100 & 75 & 150 & 6 & 0 \\
\hline 9 & 100 & 225 & 150 & 6 & 0 \\
\hline 10 & 100 & 450 & 150 & 6 & 0 \\
\hline 11 & 100 & 150 & 0 & 6 & 0 \\
\hline 12 & 100 & 150 & 75 & 6 & 0 \\
\hline 13 & 100 & 150 & 225 & 6 & 0 \\
\hline 14 & 100 & 150 & 450 & 6 & 0 \\
\hline 15 & 100 & 150 & 150 & 4 & 0 \\
\hline 16 & 100 & 150 & 150 & 5 & 0 \\
\hline 17 & 100 & 150 & 150 & 7 & 0 \\
\hline 18 & 100 & 150 & 150 & 6 & 2 \\
\hline 19 & 100 & 150 & 150 & 6 & 4 \\
\hline 20 & 100 & 150 & 150 & 6 & 8 \\
\hline 21 & 100 & 150 & 150 & 6 & 16 \\
\hline
\end{tabular}

*Dose referência. 
Após 459 dias de cultivo/condução as plantas foram cortadas, acondicionadas em sacos de papel e secas em estufa de circulação forçada de ar a $65^{\circ} \mathrm{C}$ até atingirem peso constante, o que correspondeu a três dias de secagem. Após esse período, avaliou-se a produção de massa seca (MS) da parte aérea com o auxílio de uma balança eletrônica de precisão. Os resultados foram submetidos à análise de variância e as doses das variáveis foram ajustadas a equações de regressão ao nível de $5 \%$.

\section{RESULTADOS E DISCUSSÃO}

Verificou-se que nos intervalos considerados $\left(0-400 \mathrm{mg} \mathrm{kg}^{-1}\right.$ para $\mathrm{N} ; 0-450$ $\mathrm{mg} \mathrm{kg}^{-1}$ para $\mathrm{P}$ e $\mathrm{K} ; 4$-7 para níveis de $\mathrm{pH}$ do solo e, de 0 - $16 \mathrm{Mg} \mathrm{ha}^{-1}$ para cama de aviário), as respostas foram quadráticas (Figuras 1A, 1B, 1C, 1D e 1E), o que sugere que as doses utilizadas estão dentro das exigências da espécie nas condições do experimento.

A resposta da palmeira-realaustraliana à adição de $\mathrm{N}$, isto é, o acréscimo na produção de MS (relação entre a DMET e a dose zero) foi de 10,3\% (Tabela 2). A dose de máxima eficiência técnica (DMET) foi atingida quando se adicionou $100,3 \mathrm{mg} \mathrm{kg}^{-1}$ de N. Nesta dose a quantidade de matéria seca acumulada na parte aérea foi de 47,72 g por vaso, contra 43,23 g no tratamento sem adição de $\mathrm{N}$.

Luz et al. (2006) também obtiveram resposta positiva para a aplicação de nitrogênio em palmeira-ráfia (Rhapis excelsa), com aumento da altura, número de folhas e massa seca de parte aérea. Oliveira et al. (2011) observaram que a adubação nitrogenada proporcionou um incremento significativo para taxa relativa do crescimento em altura de mudas de açaí (Euterpe oleracea).

Os resultados positivos no desenvolvimento vegetal proporcionados pelo nitrogênio estão relacionados com sua função estruturante no tecido vegetal e por constituir a maioria das enzimas responsáveis pelas reações bioquímicas das plantas.

A relação entre a produção de matéria seca e as doses de $\mathrm{P}$ adicionadas pode ser vista na Figura 1B. O acréscimo na produção de MS em função da adição de $\mathrm{P}$ foi de 75,7 \%. A importância da adubação fosfatada em solos tropicais é bem conhecida. Os baixos teores no solo associado à alta fixação com os óxidos de $\mathrm{Fe}$ e $\mathrm{Al}$, tornam este nutriente limitante aos cultivos. 

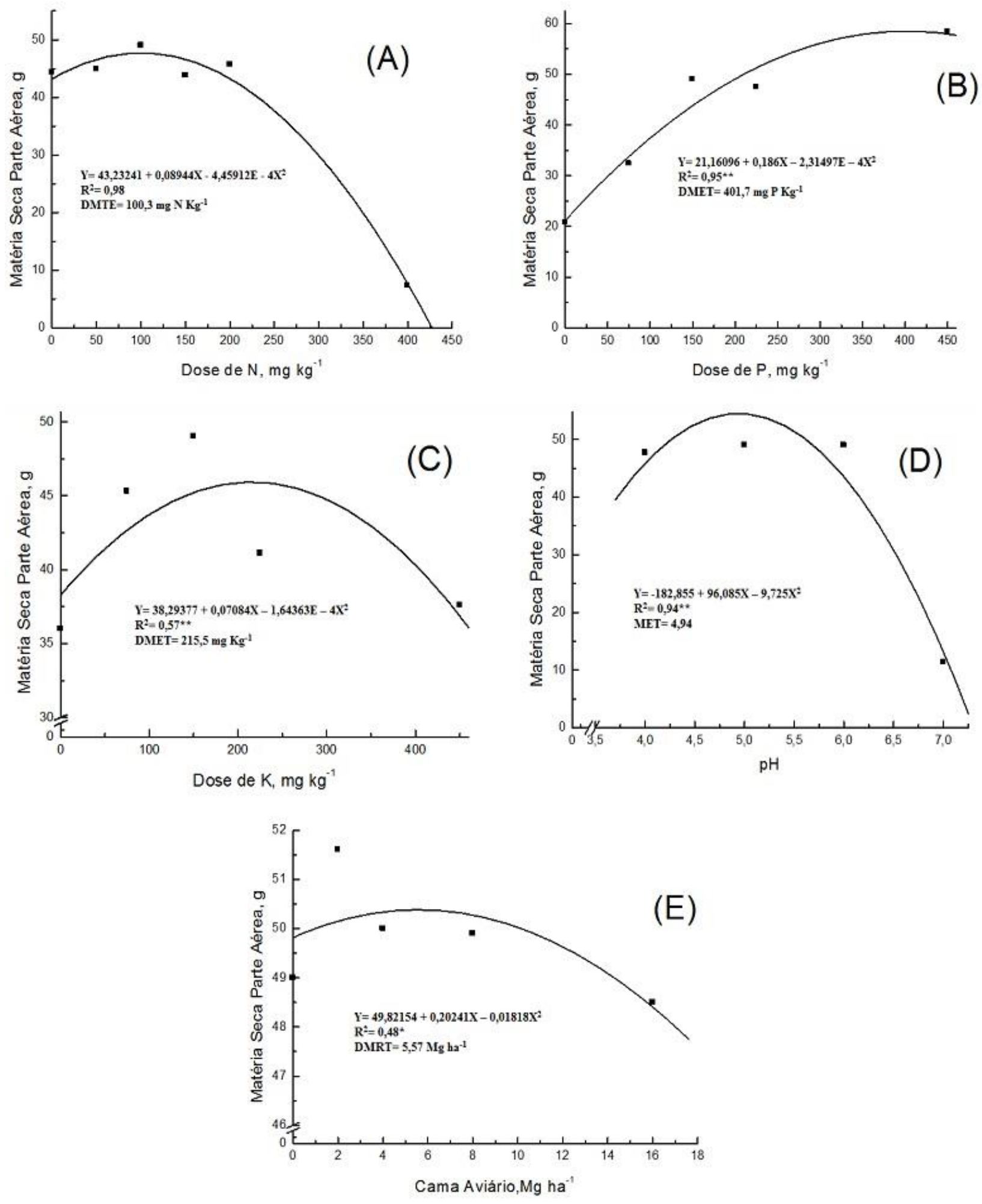

Figura 1. Produção de matéria seca da parte aérea de palmeira-real-australiana (A. alexandrae) em função de doses de Nitrogênio (A), Fósforo (B), Potássio (C), níveis de pH do solo (D) e de doses de Cama de aviário (E), Itajaí, SC, 2012. *Significativo a 5\% pelo teste F. **Significativo a $1 \%$ pelo teste $\mathrm{F}$. 
Tabela 2. Equações de regressões de matéria seca da parte aérea da palmeira-real-australiana e dose de máxima eficiência técnica (DMET) em função de doses de nitrogênio, fósforo, potássio e cama de aviário, e máxima eficiência técnica (MET) em função do pH do solo, Itajaí, SC, 2012.

\begin{tabular}{|c|c|}
\hline Tratamentos & Matéria Seca da Parte Aérea \\
\hline $\mathrm{N}$ & $\begin{array}{l}Y=43,23+0,0894 X-0,0004459 X^{2} \\
R^{2}=0,98^{*} \\
\text { DMET }=100,3 \mathrm{mg} \mathrm{N} \mathrm{kg}^{-1}\end{array}$ \\
\hline $\mathrm{P}$ & $\begin{array}{l}\mathrm{Y}=21,16+0,186 \mathrm{X}-0,000231497 \mathrm{X}^{2} \\
\mathrm{R}^{2}=0,95^{* *} \\
\mathrm{DMET}=401,7 \mathrm{mg} \mathrm{P} \mathrm{kg}^{-1}\end{array}$ \\
\hline $\mathrm{K}$ & $\begin{array}{l}\mathrm{Y}=38,29+0,07084 \mathrm{X}-0,00016436 \mathrm{X}^{2} \\
\mathrm{R}^{2}=0,57 * * \\
\mathrm{DMET}=215,5 \mathrm{mg} \mathrm{K} \mathrm{kg}^{-1}\end{array}$ \\
\hline $\mathrm{pH}$ & $\begin{array}{l}\mathrm{Y}=-182,8+96,085 \mathrm{X}-9,725 \mathrm{X}^{2} \\
\mathrm{R}=0,94 * * \\
\mathrm{MET}=4,94\end{array}$ \\
\hline CAMA & $\begin{array}{l}\mathrm{Y}=49,82+0,20241 \mathrm{X}-0,01818 \mathrm{X}^{2} \\
\mathrm{R}^{2}=0,48 * \\
\mathrm{DMET}=5,57 \mathrm{Mg} \mathrm{ha}^{-1}\end{array}$ \\
\hline
\end{tabular}
*Significativo a $5 \%$ pelo teste $\mathrm{F}$. **Significativo a $1 \%$ pelo teste $\mathrm{F}$.

Os teores iniciais de $\mathrm{P}$ no solo de 1,5 $\mathrm{mg} \mathrm{kg}^{-1}$ foram muito baixos (CQFS, 2004). Desse modo, com a aplicação de $\mathrm{P}$ houve grande incremento na produção de MS da parte aérea da palmeira-real-australiana como verificado no presente trabalho.

Luz et al. (2006) também obtiveram resultado positivo com a aplicação de $\mathrm{P}$ em palmeira-ráfia (Rhapis excelsa) obtendo aumento da altura e do diâmetro do colo.
O acréscimo de matéria seca em função da adição de $\mathrm{P}$ observado no trabalho provavelmente está relacionado com a função estruturante do elemento, o qual é constituinte dos fosfolipídios de membrana celular e, ainda, é componente dos ácidos nucleicos, nucleotídeos e coenzimas.

O acréscimo na produção de MS atribuído à adição de potássio foi, em média, de $20 \%$. Para a produção de mudas o melhor 
resultado foi obtido com a adição de $215,5 \mathrm{mg}$ de $\mathrm{K}$ por $\mathrm{kg}$ de solo. A resposta positiva à aplicação de $\mathrm{K}$ pode ter ocorrido pois, segundo Taiz \& Zeiger (2009), o potássio é requerido como cofator de mais de 40 enzimas, estabelece o turgor celular, participa da manutenção da eletroneutralidade celular e controle das células guardas do estômato.

A resposta das palmáceas/arecáceas nativas à calagem tem sido baixa, resultado da adaptação destas espécies às condições de elevada acidez dos solos tropicais em geral. Isto foi verificado também no presente trabalho; as maiores produções de MS foram obtidas nos tratamentos em que os $\mathrm{pHs}$ foram mais baixos, ao redor de 5,0 (Figura 1). Para o cultivo da pupunha recomenda-se a elevação na saturação de bases para $50 \%$, sendo que essa palmeira se desenvolve satisfatoriamente em solos ácidos, com $\mathrm{pH}$ entre 4,5 - 5,5 (MORO, 2004).

A adição de doses de cama de aviário elevou os teores de MS da parte aérea da palmeira real em apenas $1,1 \%$ em relação à dose zero. Esta baixa resposta à adição de cama pode ser atribuída ao fato de que nos tratamentos com doses de cama de aviário foi feita a adubação básica à base de NPK 100150-150 mg kg-1 e o $\mathrm{pH}$ foi corrigido para 6,0, anulando, desse modo, o efeito dessa adição.
De modo geral, a matéria orgânica no solo aumenta a capacidade de retenção de água e nutrientes, reduzindo a lixiviação e volatilização, aumenta a capacidade de troca catiônica, aumenta o poder de tamponamento, reduz a densidade, aumenta a porosidade e controla a temperatura (GUERRINI \& TRIGUEIRO, 2004; SANTOS et al., 2008; MELO \& ALLEONI, 2009) favorecendo desse modo o desenvolvimento vegetal.

Os resultados obtidos no presente trabalho mostram que a adubação fosfatada desempenha um papel fundamental na produção de mudas da palmeira-realaustraliana, sendo os ganhos com massa seca $(75,7 \%)$ superiores aos obtidos com as adubações nitrogenadas $(10,3 \%)$ e potássicas (20\%) e que as adições de doses elevadas de calcário poderão ser mais prejudiciais do que a sua omissão.

\section{CONCLUSÕES}

As doses de máxima eficiência técnica que garantiram as melhores respostas em produção de matéria seca da parte aérea de mudas de palmeira-real-australiana foram 100,3 mg de N, 401,7 mg de P e 215,5 mg de $\mathrm{K}$ por kg de solo.

A adição de cama de aves proporcionou baixo ganho de massa seca 
$(1,1 \%)$, sendo a dose de máxima eficiência técnica de 5,57 $\mathrm{Mg} \mathrm{ha}^{-1}$.

$\mathrm{O}$ pH ideal para a produção de mudas de palmeira-real-australiana foi de 4,94.

\section{AGRADECIMENTOS}

Os autores agradecem à Fundação de Apoio à Pesquisa de Santa Catariana pelo financiamento da pesquisa.

\section{REFERÊNCIAS}

ANGHINONI, I.; BAYER, C. 2004. Manejo da fertilidade do solo. In: BISSANI, C.A.; GIANELLO, C.; TEDESCO, M.J.; CAMARGO, F.A.O., ed. Fertilidade dos solos e manejo da adubação de culturas. Porto Alegre, Gênesis. p.252-264.

BISSANI, C.A.; GIANELLO, C. CAMARGO, F.A.O.; TEDESCO, M.J. 2008. Fertilidade dos solos e manejo da adubação de culturas. Porto Alegre: Metrópole, $2^{\mathrm{a}}$ ed. 343p.

CQFS-RS/SC. 2004. Manual de adubação e calagem para os Estados do Rio Grande do Sul e de Santa Catarina. Porto Alegre: SBCS, $10^{a}$ ed. 400p.

GUERRINI, I.A.; TRIGUEIRO, R.M. 2004. Physical and chemical attributes of substrates composed of biosolids and carbonized rice chaff. Revista Brasileira de Ciência do Solo, Viçosa, v.28, n.1, p.1069-1076.

LUZ, P.B.; TAVARES, A.R.; PAIVA, P.D.O.; MASSOLI, L.A.L.; AGUIAR, F.F.A.; KANASHIRO, S.; STANCATO, G.C.; LANDGRAF, P.R.C. 2006. Efeitos de nitrogênio, fósforo e potássio no crescimento de Rhapis excelsa (thunberg) henry ex. rehder (palmeira-ráfia). Ciência e Agrotecnologia, Lavras, v.30, n.3, p. 429-434.

MELO, V.F.; ALLEONI, L.R.F. (Ed.). Química e mineralogia do solo: parte 2 - aplicações. $1^{\text {a }}$ ed., Viçosa - MG, Sociedade Brasileira de Ciência do Solo, 2009. 685p.

MORO, J.R. A Cultura da pupunha para produção de palmito. In: SANTOS, A.F. $O$ agronegócio da pupunha e palmeira real. Embrapa, Documentos 105, 2004. $163 p$.

OLIVEIRA, C.J.; PEREIRA, W.E.; MESQUITA, F.O.; MEDEIROS, J.S.; ALVES, A.S. 2011. Crescimento inicial de mudas de açaizeiro em resposta a doses de nitrogênio e potássio. Revista Verde, Mossoró, v.6, n.2, p.227 - 237.

RODRIGUES, A.S.; DURIGAN, M.E. 2007. $O$ agronegócio do palmito no Brasil. Londrina: IAPAR. 131p.

RODRIGUES, A.S; DURIGAN, M.E. 2008. $\mathrm{O}$ agronegócio do palmito no Brasil. In SANTOS, A.F.; CORRÊA JÚNIOR, C.; NEVES, E.J.M. Palmeiras para produção de palmito: juçara, pupunheira e palmeira real. Colombo: Embrapa Florestas.p.15-21.

Recebido em: 27/11/2015 Aceito para publicação em: 18/11/2015 